\title{
PROBLEMS WHEN EVALUATING THE ACHIEVEMENTS OF THE PUPILS' MOTOR EDUCATION IN THE PRIMARY SCHOOL /THE PHYSICAL EDUCATION AND SPORT MARKS - FEEDBACK, MOTIVATION, STRESS OR SIMPLY NUMBERS FOR THE BULGARIAN PUPILS/
}

\author{
V. Ivanova* \\ Faculty of Education, Trakia University, Stara Zagora, Bulgaria
}

\begin{abstract}
The present report is focused on the control, the check and the assessment as obligatory elements of the motor education in the Bulgarian school. Criteria and indexes making objective the scholar achievements evaluation of the primary school pupils are discussed. An assessment model applied in a school in Cyncoed (Cardiff Wales) is presented as a good practice attempt and example.
\end{abstract}

Key words: primary school age, physical education and sport, scholar achievements, evaluation

\section{INTRODUCTION}

The bad and the good evaluations of the human activity are a part of the life of everyone (1). The evaluations made by the adults, the other children and the child himself are an obligatory element of the motor education in the Bulgarian school (2). On the face of it the innocent numbers presenting the evaluation of the knowledge, the motor skills and relations provoke different emotions among the pupils and often discussions such as the following example: Are you "for" or "against" the digital marks of the physical education in the school? Is it the useful feedback executing the general functions of the educational process and the specific ones in the field of physical education, as follows?:

- diagnostic - the prognosis of the trainee's development on the basis of his achievements during the scholar process;

- didactic - the possibilities of making analyses, additions, sistematizations, corrections of the made mistakes in motor actions based on the acquired knolwledge, motor skills and habits;

- educational - the formation of positive personal qualities when demonstrating the own possibilities and achivements;

- stimulating - the motivation of more profound cognitive and motor activity determinig the resultant effect;

\footnotetext{
*Correspondence to: Veselina Georgieva Ivanova, Faculty of Education, Trakia University - Stara, Zagora,Bulgaria,v_g_chipeva@abv.bg
}

- regulating - as a feedback which according to a prognostic study and analysis made by the pedagogue provides a current and in perspective optimal management of the scholar and educational process (3).

\section{A trivial story with a lesson}

A football match. A lot of fans and an important derby. The coach $X$ changes the player Y. During the change:

1. The audience hisses the player

Cause: The footballer did not play well $=\mathrm{a}$ negative feedback. Conclusion: $\mathrm{He}$ understands that he has to train more in order to be more successful in the future football matches.

2. The audience applauds the player

Cause: The footballer played well $=$ a positive feedback. Conclusion: The efforts made in the process of training are resultant but he should continue to work hard in the future, too.

The effort, the evaluation and the knowledge are important factors of the scholar life (4). A part of the trainees make efforts to receive good marks, others - to acquire knowledge and skills and thirds - to acquire knowledge and skills in order to receive good marks. But it is an uncontestable fact that the numerous digits written down by the teacher in different diaries and documents do not reflect the unique capacities and talents. For some pupils they are a cause of happiness and thankfulness, for others - of stress and for third ones - simply digits necessary to pass to a higher class. More often the children perceive them as a factor but 
not as a means stimulating the trip toward the knowledge. The satisfying erudition orientates toward a personal self-improvement (5).

\section{How the pupils' achievements in the field of motor education in Bulgaria are assessed?}

The evaluation is the final stage of the act of the control activity which is executed in the beginning of a determined class education, before or after new parts /kernels/ or topics of the scholar content, at the end of the scholar term and the scholar year. This check is realized by different methods and means depending on the trainees' age and training, on the motor actions nature and on the physical exercises. In order to be stimulating and educating the evaluation should be real, i.e. objective and just, it should be clear and motivated on an individual or group plane and differentiated according to the different parts of the pupils' training /physical, technical, tactical, psychical and theoretical/. The verbal evaluation in the form of an assessing judgement /"Perfect", Bravo, I am content with the execution", "I am not content, the result is very poor" etc./ by which the quality of the motor task execution is assessed, especially when working with smaller pupils or the written digital mark together with the respective verbal equivalent are used.

The evaluation of the scholar subject "Physical education and sport" is more complex. The checked objects in the primary school are the following:

- Motor culture /motorics/ of the trainees expressed through the acquired motor skills and habits for a determined period of time. The techique and the quantitative result during the fulfillment of the planned programm educational content for the determined class are checked. The evaluation is qualitative /for the technique/ and quantitative /for the achieved result/ during the physical exercises execution and it is according to the state educational requirements /standards/ for the determined class.

- Physical active capability. The degree of the motor qualities development is checked periodically measured by different tests. The evaluation is made in the form of points and digits determining the complex development of the conditional and coordinative capacities of the organism and it is reglamentated in the program documents for the primary school age.

-Knowledge. In the filed of physical education the volume and the degree of the acquired new and main notions are assessed; the technique and the tactics when executing and applying the physical exercises; help and keeping, organisation and self-organisation.

-Activity of the pupil in the process of the motor education and his practical skills for applying the studied physical exercises under playing, competing, sport and life conditions. An important part is the evaluation of the trainee's activity and competences - by verbal stimulating judgments or by an assessment mark in points, as it is recommended in the initial stage of the main educational degree /up to 30 points of the general complex evaluation of 150 points/.

- Physical development. It is checked in the beginning and at the end of the scholar year on the basis of anthropometric measurements according to morphological and physiometrical indexes /hight in a standing position, body weight, chest measurement in a pause, maximal inspiration, maximal expiration etc./. The results are assessed according to national normative tables and scales and they are written down in the personal healt file of the examined person but they are not included in the final mark of physical education.

In the sportive pedagogical practice the following methods apply most often in order to check and assess the motor education results: control execution, pedagogical observation, motor testing, normatives covering, oral examination, somatometry.

Through the control execution of a determined exercise or a combination of exercises the degree of a determined motor action mastering and the quality of its execution by each pupil is assessed. The teacher observes visually every pupil and as a specialist evaluates him expertly on the basis of the technical parameters of the executed motion or physical exercise and the committed mistakes. The possible mark is in 5 points as follows:

- excellent- the exercise is executed without mistakes, with fleetness;

- very good - the mistakes are insignificant related to the technique details;

- good - the exercise is executed with mistakes but they do not influence the form and the structure of the whole motion;

- satisfactory - the execution is made in general but with grave mistakes;

- poor - the exercise can not be executed.

In the physical education teacher's books and in other specialized sources the deviations from the correct technical execution of the physical execises provided in the scholar content for the determined age are indicated /most often made mistakes and the methodology for their correction/ but there is not a differentiated presentation according to 
the 5-point scale used in the practice. The primary teacher /who in Bulagria teaches many subjects and who is not a physical education specialist/ should determine whether the mistakes are important or not knowing that only when the marks are objective they may be an indicator of the trainee's results and a prognostic index of the scholar process. But there are many studies which show negative tendencies in the children's motorics - a function of the physical and technical training made during that important period of the children's development.

In such an aspect an experiment was made with students from the $3^{\text {rd }}$ course in the specialty of "Primary school pedagogy with foreign language" who have passed a lecture course of "Theory and methodology of physical education in the $1^{\text {st }}-4^{\text {th }}$ classes". The students have been acquainted with the criteria elaborated by a team of primary teachers and teachers - specialists of physical education in a school in Cyncoed /Cardiff, Wales/ in order to assess the achievements of 6-year old pupils in the six groups of motor activities: dances, games, gymnastics, invasive games, games with netting and games in the open air through which the motor education is realized (6).

\section{Evaluation of the physical education and sports}

Dances

Pupil's name:

Age 6

Class:

Teacher:

Date of evaluation:

execution: Yes / No

To work alone creatively and with imagination and with his partner or in a group to create motifs or structures /a combination of movements/ of easy dances ..........

To dance expressively and with feeling .........

To dance smoothly and with control ...........

To limber up in the beginning and to lead independently his body to repose /to relax/ after finishing ...

To understand how the dances contribute to the health

To use appropriate criteria for evaluation and improvement of the quality of work - his own and of the others

To speak about the dances with understanding using an appropriate language and terminology

Self-evaluation /for example his own capacities and forces and suggestions for amelioration/ ...........

What follows? / Individual needs

Games

Pupil's name:
Age 6 Class:

IVANOVA $V$

Teacher:

Date of evaluation:

.execution: Yes / No

To use main skills as passes below, rolling or tapping ...........

To do sometimes passes above ..........

To meet, receive or stop with confidence a passed medium size ball ..........

To catch sometimes a flying medium size ball

To follow a moving ball or other apparatus passed toward him / her

To throw, hit or kick by different ways according to the game requirements ..........

To choose different ways of ball hitting, throwing or kicking ..........

To decide where to stay in order to make the play difficult for the adversary ..........

To describe what he and the others do ..........

To describe what his body feels during the game ..........

Self-evaluation: /for example what is the most / the least agreeable for him /

What follows? / Individual needs

Gymnastics

Pupil's name:

Age 6

Class: ...........

Teacher:

Date of evaluation:

execution: Yes / No

To execute during a long time a series of more complex gymnastic exercises and to make combinations of them ..........

To chose, apply /exercise/ and improve series of exercises at his discretion ..........

To determine when the movements are correctly executed

To show exercises for the big muscular groups

To determine which joint/s/ is influenced

To indicate ways for improvement of the series and to accentuate one of those ways during his work ...

To be the leader when working in a small group ..........

To adapt the approaches in order to involve all group members

Self-evaluation /for example his own capacities and forces and suggestions for their improvement/...

What follows/ / Individual needs

Invasive games

Pupil's name:

Teacher: ..........

Date of evaluation: .execution: Yes / No 
To play effectively with the necessary speed and precision in small as well as in bigger teams

To decide quickly where and when to pass the ball showing a good sense of orientation for the situation ...........

To change his tactics and to adapt his skills to the game situation ..........

To play bigger range of games using different skills ..........

To lead the limbering up of small groups

To know why the application of tactic interaction is important in the game ..........

Self-evaluation /for example his own capacities and forces and suggestions for their improvement/ ...

What follows/ / Individual needs ..........

When making the students acquainted with this good practice example we put the accent on the details of the requirements concerning the technique, the tactics, the psychic, the esthetics etc. of the suggested motor units. The case the students had to solve was limited in the elaboration of criteria permitting the objectivism of the technical execution of main motor units of the physical education scholar content for the $1^{\text {st }}$ class, kernel "Track-andfield athletics" /long jump from place with two legs, race in a straight line, dense ball throw with two arms above the head/. The results were poor - few of the participants in the experiment were satisfactory. When making a comparative analysis of the marks received during the semester examination of the subject /mean group's result/ and the marks received during the experiment a big difference between them was shown.

Such practical scholar tasks applied in a system could develop the students' skills to give the correct indicators but the following question arises: Isn't it that burden too heavy for the primary teacher? Isn't it time that the specialist assumes his responsibility for the motor training, the development and the education of our children?

The control execution is applied also when measuring the quantitative result /each trainee's achievement/. For example: the time necessary to run a determined distance $-50 \mathrm{~m}$ /in sec/ or the direct hits in the sportive games /number/ etc. During such measurable motor activities the evaluation is much more objective because the quantitative result is a function of the technique and represents the degree of the execution quality.

In order to activate the participants in the scholar process and to achieve bigger results it is necessary to create competitive conditions - running and jumping by pairs or in series, handicap race /priority/, relay-race and competitive games etc.

The pedagogical observance is applied at each stage of the training. Through it the teacher realizes the current control in the motor education process by surveilling, analyzing and prognosticating it and the trainees' results. Besides during the control execution the visual observation is applied also during such motor activities which do not permit every pupil to execute the determined exercise independently such as the games - movable, relay-race, sportive and preparative, sportive etc. During the game the teacher observes the technical and tactical children's execution as well as their capacity to apply the learnt elements and tactical decisions when there are suddenly changing game conditions, i.e. variably, originally and creatively. During the control lessons or competitive games the teacher evaluates determined qualitative indexes of the sportive technique and gives marks $/ 15$ or 20 points/ in order to make the terminal and annual Physical Education and Sport evaluations.

The motor testing is the main method for control and evaluation of the development level of the main motor qualities. By executing standard motor tasks /motor tests/ and analyzing the achieved quantitative results one may get a real idea of the physical active capability and the level of each person and of all trainees as a whole. This method is applied in the beginning and at the end of the scholar year /or at the end of the first scholar term/ in the form of test batteries determined for the different age groups in the program documents. For the $1^{\text {st }}-4^{\text {th }}$ class pupils the test battery includes 4 tests /50 meters dash race, long standing jump with two legs, dense ball throw with two hands above the head, 200 meters shuttle race/ through which the level of the trainees'condition capacities are determined

The test results are transformed into point marks on the basis of specially elaborated /up to 25 points for each test/ age and sex evaluation tables. The points sum received for each motor quality gives an idea of the level of the physical active capability and it represents a complex result which is transformed into a digital or oral mark. For example: in the $1^{\text {st }}$ $4^{\text {th }}$ class the maximal points which the pupil may receive for the four tests are 100. They may be transformed into a digital mark of the physical active capability as follows: excellent - from 100 to 81 points, very good - from 80 to 61 points, good - from 60 to 41 points, 
satisfactory - from 40 to 21 points and poor from 20 to 0 points.

Norms covering. Through this method the teacher is not interested in the precise achievement of the pupil but in the execution of the norm determined for the given age and sex for some motor action /for example $300 \mathrm{~cm}$ ball throw/. The evaluation in this case is "executed" or "non executed".

Oral exam and conversation are main methods for control and evaluation of the trainees' theoretical knowledge related to a concrete motor activity realized by them. Through the conversation a direct contact between the two main subjects of the training/teacher - pupil/ is realized and an oral examination is made according to the given theoretical or practical problem. This method contributes to the logical thinking, the verbal and public expression skill, the embarrassment and fear overcoming and gives possibilities of the trainee's individual features revealing /thinking, memory, emotional state, selfevaluation and self-control/ etc. In the primary school the oral exam should not stress the children but should predispose and stimulate them to a more active mental and cognitive and system motor activity.

Somatometry is one of the main methods of the body and its parts measurment according to determined anthropometric, somatoscopic and physiometric indexes. By using approved evaluation tables, indexes and scales the constitution harmony and the personal physical development level are evaluated. It is made in the beginning and at the end of the scholar year by a qualified medical person in the presence of the pedagogue. The physical development results and evaluations are not a component of the physical education mark but they should be obligatorily communicated to the parents and to the children themselves /especially the bigger pupils/. The summarized evaluations of the trainees' helath status and physical development are reported also to the pedagogical councils of the educational institutions.

As a method the oral evaluation is used in the $1^{\text {st }}$ class. It is applied in some moments in order to assess the motor education achievement.

The rating evaluation gives a digital expression of the pupils' achievements according to the different parts of their motor training - technical, physical, theoretical, tactical and psyshic.
The Physical education and Sport mark in the $1^{\text {st }}-4^{\text {th }}$ class is formed on the basis of three main indicators:

I. Execution quality. For the correct thecnical execution of the studied motor actions the pupil may receive a maximal number of points -20 ;

II. Quantitative result. For an achieved level of physical active capability - a maximal number of 100 points /4 tests - 25 points each one/;

III. Qualitative and quantitative increase. For a shown positive attitude and made efforts maximum 30 points.

The received point marks /up to 150 points maximum/ are transformed into the final rating evaluation according to the 5-stage scale:

- unsatisfactory - Poor $/ 2 /$ - from 0 to 50 points

- satisfacory - Satisfactory /3/ - from 51 to 75 points

- good - Good /4/ - from 76 to 100 points

- very good - Very Good /5/ - from 101 to 125 points

- excellent - Excellent /6/ - over 126 points.

\section{CONCLUSION}

The educational and training function of the evaluation is achieved only if it is objective, communicated and analyzed together with the trainees. By this way the creation of real selfevaluation and self-control skills are stimulated. In this context we have submitted for discussion the possibility of including the motor testing and physical development results in the scholar portfolio in order to enlarge the possibilities not only of the teachers but also of the parents and the children themselves of following the progress, the weak and strong sides and the talent.

\section{REFERENCES}

1. Andreev, M. The process of learning. Didactics. Sofia, University Publishing House St. Kl. Ohridski, pp. 247-248, 2001.

2. Boeva, B., V. Ivanova. Theory and methodology of physical education. A textbook for students of the Trakia University. Theoretical and methodological bases of physical education. Part one. Stara Zagora, pp. 143-148, 2012.

3. Radev, Pl. and coll. Pedagogy. Plovdiv, Hermes publishing, pp. 505-507, 2007.

4. Radev, Pl. General school subject. Plovdiv, University Publishing House Paisii Hilendarski , 2005.

5. Teneva, M. Master of didactic topics of students. The first part. Stara Zagora, pp. 202-230, 2012.

6. Peneva, B. School physical education and sport in Europe - situation and trends at the beginning of the XXI century. Avantgard Prima publishing, Sofia, pp. 39-46, 2011. 